\title{
Mutation analysis of BRCAI/2 mutations with special reference to polymorphic SNPs in Indian breast cancer patients
}

This article was published in the following Dove Press journal:

The Application of Clinical Genetics

\author{
Nidhi D Shah' \\ Parth S Shah ${ }^{2}$ \\ Yash Y Panchal ${ }^{3}$ \\ Kalpesh H Katudia ${ }^{3}$ \\ Nikunj B Khatri ${ }^{3}$ \\ Hari Shankar P Ray ${ }^{3}$ \\ Upti R Bhatiya ${ }^{3}$ \\ Sandip C Shah ${ }^{3}$ \\ Bhavini S Shah' \\ Mandava V Rao ${ }^{4}$ \\ 'Department of Pediatrics, Nassau \\ University Medical Center, New \\ York City, NY, USA; ${ }^{2}$ Department of \\ Medicine, Lahey Hospital and Medical \\ Center, Boston, MA, USA; ${ }^{3}$ Supratech \\ Micropath Laboratory and Research \\ Institute, Ahmedabad, Gujarat, \\ India; ${ }^{4}$ School of Sciences, Gujarat \\ University, Ahmedabad, Gujarat, India
}

Correspondence: Sandip C Shah Supratech Micropath Laboratory and Research Institute, Ahmedabad, Gujarat 380006, India

Tel +9l 9824030580

Email supratech18@gmail.com
Background: Germline mutations BRCA1 and BRCA2 contribute almost equally in the causation of breast cancer (BC). The type of mutations in the Indian population that cause this condition is largely unknown.

Purpose: In this cohort, 79 randomized BC patients were screened for various types of BRCA1 and BRCA2 mutations including frameshift, nonsense, missense, in-frame and splice site types. Materials and methods: The purified extracted DNA of each referral patient was subjected to Sanger gene sequencing using Codon Code Analyzer and Mutation Surveyor and next-generation sequencing (NGS) methods with Ion torrent software, after appropriate care.

Results: The data revealed that 35 cases were positive for BRCA1 or BRCA2 (35/79: 44.3\%). BRCA2 mutations were higher (52.4\%) than BRCA1 mutations (47.6\%). Five novel mutations detected in this study were p.pro163 frameshift, p.asn997 frameshift, p.ser148 frameshift and two splice site single-nucleotide polymorphisms (SNPs). Additionally, four nonsense and one in-frame deletion were identified, which all seemed to be pathogenic. Polymorphic SNPs contributed the highest percentage of mutations (72/82: 87.8\%) and contributed to pathogenic, likely pathogenic, likely benign, benign and variant of unknown significance (VUS). Young age groups (20-60 years) had a high frequency of germline mutations $(62 / 82 ; 75.6 \%)$ in the Indian population. Conclusion: This study suggested that polymorphic SNPs contributed a high percentage of mutations along with five novel types. Younger age groups are prone to having $\mathrm{BC}$ with a higher mutational rate. Furthermore, the SNPs detected in exons 10, 11 and 16 of BRCA1 and BRCA2 were higher than those in other exons 2, 3 and 9 polymorphic sites in two germline genes. These may be contributory for $\mathrm{BC}$ although missense types are known to be susceptible for cancer depending on the type of amino acid replaced in the protein and associated with pathologic events. Accordingly, appropriate counseling and treatment may be suggested.

Keywords: breast cancer, BRCA1 and BRCA2 gene mutations, polymorphic SNPs, novel types, age, NGS, Sanger gene sequencer

\section{Introduction}

The continuing global demographic and epidemiologic transitions signal an everincreasing cancer burden over the next decades, particularly in low- and middle-income countries (LIMC), with over 20 million new cancer cases expected annually as early as 2025. Breast cancer (BC) is one of the most common and dreadful diseases affecting Indian women. ${ }^{1,2}$ It is actually considered the main type of cancer among new cases in India. ${ }^{3}$ It has been divided into two subtypes, early-onset and late-onset, based on agespecific cancers. Mutation in BRCA1 is thought to account for about $45 \%$ of families, whose locus is to chromosome $17 \mathrm{p}$. The second one is mapped to chromosome $13 \mathrm{q}$ and 
responsible for the early onset of BC. ${ }^{4}$ About 200 mutations in both genes have been reported and are widely scattered across them..$^{5-7}$ Most mutations of these genes affect the structure and function of the gene, altering protein synthesis and function (34\% BRCA1 and 38\% BRCA2 mutations), and others are missense types that alter amino acids but do not truncate the protein. ${ }^{8,9}$ Hence, the mutation of BRCA1/2 results in the gene's function silencing or over-activation.

According to the GLOBOCAN project set up by the International Agency for Research on Cancer (IARC), ${ }^{10}$ 145,000 new cases were diagnosed and 70,000 deaths occurred in India in 2012. Considering the size of the population, the incidence of BC in India appears to be lower, when compared to developed countries such as the USA (233,000 cases) and the European union $(362,000$ cases $)$. But the ratio of death in India was much higher (48.3\%) in comparison with the USA $(18.9 \%)$ and the European union $(25.4 \%))^{11,12}$ Thus, the frequency of BC patients seems to be lower than in the developing countries, but mortality is higher in our country attributable to lifestyle changes, delay in introduction of cancer screening centers and limited medical awareness including treatment. ${ }^{12-14}$

The relatively high death rate from $\mathrm{BC}$ in the Indian health care system thus demonstrates a need for a cost-effective method of early detection, screening and treatment. ${ }^{12}$ However, early detection of hereditary $\mathrm{BC}$ is essential to reduce this type of cancer in a developing country like India. Hence, this study was undertaken to detect the germline mutations using molecular diagnostic methods and their correlation with pathogenic events for its better treatment in addition to other factors, such as age, in our cohort. Emphasis is also paid to the involvement of missense mutations occurring in BRCA1 and BRCA2 genes of BC patients in the Indian population.

\section{Materials and methods Patient selection}

A total of 79 referral, diagnosed, pre- and post-menopausal $\mathrm{BC}$ cases from India were selected. EDTA blood samples and a request for biological specimens were collected from the respondents after completion of consent forms and counseling. The patients provided test reports which were used for our current research. This work was approved by Gujarat University (Ahmedabad, India) Human Ethical Committee (GUHEC/001/2015) for investigation.

\section{DNA extraction}

Genomic DNA was extracted from 3-4 mL of peripheral EDTA blood using automated DNA extraction method
(PerkinElmer Inc., Waltham, MA, USA). The concentration of DNA was determined using the Qubit 2.0 Fluorometer (Thermo Fisher Scientific, Waltham, MA, USA).

\section{Targeted next-generation sequencing (NGS) (amplification)}

For targeted NGS analysis, the Ion AmpliSeq ${ }^{\text {TM }}$ BRCA1 and BRCA2 Panel (Thermo Fisher Scientific) containing 167 primer pairs in three pools (Pool 1 is 56 pairs, 2 is 56 pairs and 3 is 55 pairs) was used. Multiplex PCR was performed using 10-30 ng genomic DNA with a premixed primer pool and Ion AmpliSeq ${ }^{\mathrm{TM}} \mathrm{HiFi}$ master mix; Ion AmpliSeq ${ }^{\mathrm{TM}}$ Library Kit 2.0; Cat. no. 4480441) for $2 \mathrm{~min}$ at $99^{\circ} \mathrm{C}$, followed by 19 cycles of denaturation at $99^{\circ} \mathrm{C}$ for $15 \mathrm{~s}$ and annealing and elongation at $60^{\circ} \mathrm{C}$ for $4 \mathrm{~min}$, ending with a holding period at $10^{\circ} \mathrm{C}$. The PCR amplicons were treated with $2 \mu \mathrm{L}$ FuPa reagent to partially digest primer sequences and phosphorylate the amplicons at $50^{\circ} \mathrm{C}$ for $10 \mathrm{~min}$, followed by $55^{\circ} \mathrm{C}$ for $10 \mathrm{~min}$, then $60^{\circ} \mathrm{C}$ for $20 \mathrm{~min}$. The amplicons were ligated to adapters with the diluted barcodes of the Ion Xpress ${ }^{\mathrm{TM}}$ Barcode Adapters kit (Thermo Fisher Scientific) for $30 \mathrm{~min}$ at $22^{\circ} \mathrm{C}$, then $72^{\circ} \mathrm{C}$ for $10 \mathrm{~min}$. Adaptor-ligated amplicon libraries were purified using Agencourt AMPure XP (Cat no. A63881) reagents (Beckman Coulter, CA, USA). The library concentration was determined using an Ion Library Quantitation Kit (Cat no. 4468802) (Thermo Fisher Scientific), then each library was diluted to $4-8 \mathrm{pmol} / \mathrm{L}$ and the same amount of libraries was pooled for one sequence reaction. Next, emulsion PCR was carried out using the Ion OneTouch ${ }^{\mathrm{TM}} 2.0$ System and Ion PGM ${ }^{\mathrm{TM}} \mathrm{Hi}-\mathrm{Q}^{\mathrm{TM}}$ OT2 (Cat no. A27743) reagents, or Ion $540^{\mathrm{TM}}$ OT2 (Cat no. A27752) reagents (Thermo Fisher Scientific), according to the manufacturer's instructions. Template-positive Ion Sphere ${ }^{\mathrm{TM}}$ particles were then enriched with Dynabeads MyOne ${ }^{\mathrm{TM}}$ Streptavidin C1 Beads (Cat no. 65001) (Thermo Fisher Scientific) using an Ion OneTouch $^{\text {TM }}$ ES system (Thermo Fisher Scientific). Purified Ion Sphere particles were loaded on any of chip types 314, 316, or 318 (Compatible with Ion PGM) or 520, 530, or 540 (Compatible with Ion S5 System) Chip. Massively parallel sequencing was carried out on a Personal Genome Machine (PGM) sequencer (Ion Torrent ${ }^{\mathrm{TM}}$ ) using the Ion $\mathrm{PGM}^{\mathrm{TM}} \mathrm{Hi}-\mathrm{Q}^{\mathrm{TM}}$ Sequencing Kit or Ion S5 sequencer (Thermo Fisher Scientific) using Ion S5 Sequencing kit according to the manufacturer's instructions. Sequencing was performed using 500 flow runs that generated $\sim 200 \mathrm{bp}$ reads. 


\section{Sanger DNA sequencing}

Possible high confidence variants were confirmed by Sanger sequencing. PCR primers were designed using the OligoCalc (http://biotools.nubic.northwestern.edu/OligoCalc.html) to determine the properties of designed primers and Primer Blast (https://www.ncbi.nlm.nih.gov/tools/primer-blast/) was also used to check whether our region of interest was covered. Primers were synthesized at $100 \mathrm{nM}$ scale and cartridge purified (Eurofins, Bengaluru, India). PCR amplification was carried out using Takara Taq ${ }^{\mathrm{TM}}$ Hot Start Version 2.0 PCR mix with each primer at $10 \mathrm{pM}$ concentration under standard conditions. These PCR amplifications were all carried out in duplicate along with a known normal control cell line sample. Successful PCR products were purified prior to sequencing using ExoSAP-IT (Cat no. 78201) reagent (Thermo Fisher Scientific) according to the manufacturer's protocol. Purified PCR products were subjected to Sanger sequencing technique in both forward and reverse orientations with the same primer sequences used for PCR at 10 pM final concentration using BigDye ${ }^{\circledR}$ v3.1(Cat no. 4336917) according to the manufacturer's cycling conditions. BigDye ${ }^{\circledR}$ v3.1 sequencing reactions were then purified using EDTA (Cat no. 194822) and sodium acetate (Cat no. 567418) according to the manufacturer's protocol and analyzed on 3500 Genetic Analyzer (Thermo Fisher Scientific). Sanger sequencing data were analyzed using Codon Code Aligner v5.0.2, (CodonCode Corporation, Centerville, MA, USA) and Mutation Surveyor v5.0, (SoftGenetics, State College, PA, USA).

\section{Data analysis}

The sequence data were processed using standard Ion Torrent Suite ${ }^{\mathrm{TM}}$ Software running on the Torrent Server (Thermo Fisher Scientific). Raw signal data were analyzed using Torrent Suite ${ }^{\mathrm{TM}}$ version 4.4, 4.6 or 5.0. The pipeline included signaling processing, base calling, quality score assignment, adapter trimming, PCR duplicate removal, read alignment to human genome 19 reference (hg19), quality control of mapping quality, coverage analysis, and variant calling. Coverage analysis and variant calling used Torrent Variant Caller plugin software (Version $4.4,4.6$ or 5.0) in the Torrent Server. The variant caller parameter setting was germline PGM high stringency. Following data analysis, annotation of single-nucleotide variants, insertions, deletions, and splice site alterations were performed by the Ion Reporter ${ }^{\mathrm{TM}}$ Server System (Thermo Fisher Scientific), which identified nonsynonymous mutations. Splice site alteration were analyzed 2 bp upstream or downstream of exon-intron boundaries. Sequence data were visually confirmed with the Integrative Genomics Viewer (IGV) and any sequence, alignment, or variant call error artifacts were discarded. Nonsynonymous mutations were annotated using the Breast Cancer Information Core (BIC) database (https://research. nhgri.nih.gov/projects/bic/index.shtml) and ClinVar (http:// www.ncbi.nlm.nih.gov/clinvar/). ${ }^{14}$ Minor allele frequency was determined from the 1000 Genomes Project database..$^{15}$ The 5000 Exome project (http://evs.gs.washington.edu/EVS/) and The Human Genetic Variation Database (http://www. genome.med.kyoto-u.ac.jp/SnpDB) were used to identify the pathogenic conditions of the BRCA1/2 gene mutations.

\section{Statistics}

Percentage was calculated wherever necessary.

\section{Results}

After analyzing 79 referral cases of women ranging in age between 23 and 72 years, 35 BC-positive (44.30\%) cases were detected with germline gene mutations. Among them, 11 cases were with BRCA1 gene mutations (31.4\%) followed by 15 with BRCA2 gene mutations (42.9\%), and both BRCA1 and BRCA2 mutations were found in nine cases (25.7\%) (Figure 1).

\section{Mutation types in BRCAI and BRCA2}

A total of 82 mutations, 39 with BRCA1 (39/82; 47.6\%) and 43 with BRCA2 (43/82; 52.4\%), were identified in 35 cancer patients. A total of $72(72 / 82)$ missense mutations $(87.8 \%)$ with amino acid replacement (BRCA1, 36/82, $43.9 \%$; BRCA2, 36/82, 43.9\%) were detected with two novel mutations (BRCA1; two splice sites). Among other types, five frameshift mutations $(5 / 82: 6.1 \%)$ were detected in five cases (exon 1, exon 14 in BRCA1; exon 6, exon 10, exon 11 in BRCA2), where three were novel types. Others were four nonsense mutations of BRCA2 (4/82:4.9\%) and

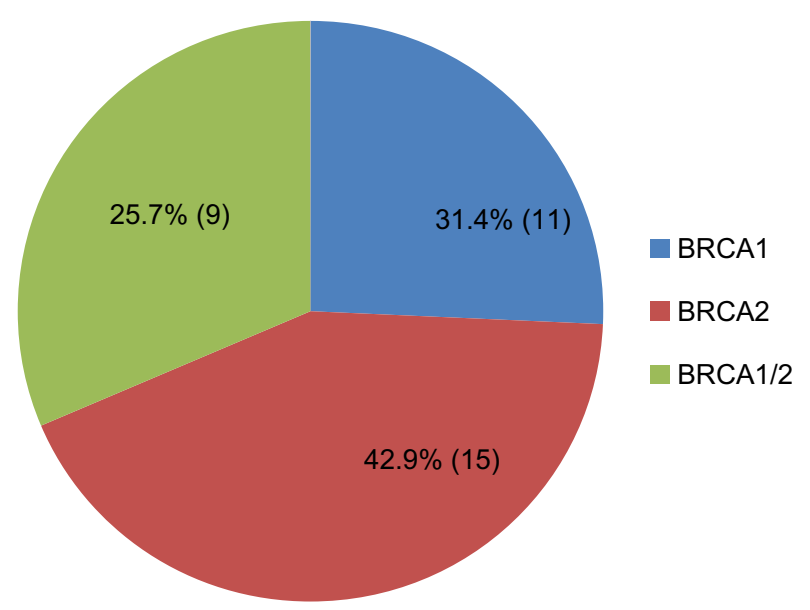

Figure I Patients $(\mathrm{n}=35)$ with BRCAI and BRCA2 mutations. 
one in-frame deletion of BRCA1 (1/82:1.2\%). A total of five novel mutations were present with three frameshift and two missense (splice site) mutations in our study (Table 1; Figure 2). Moreover, familial (20) and sporadic (15) cases were not significantly different (Table 1).

\section{Frequency of SNPs in BRCAI and $B R C A 2$ and pathologic variants}

The single-nucleotide polymorphism (SNP) frequency was higher in exons 10, 11 and 16 of BRCA1 and BRCA2 followed by exons 9, 2 and 3. In BRCA1, exon 9 had two splice site SNPs which were pathogenic and one SNP likely benign (total of three). Exon 10 had seven SNPs benign, two each with likely pathogenic and variant of unknown significance (VUS) (total of four). Exon 11 contributed 12 SNPs, each with two amino acid changes showing likely benign and two with VUS having two amino acid changes. The exon 16 possessed six benign and two with VUS. In BRCA2, exon 10 showed nine SNPs likely benign/benign/likely pathogenic, four SNPs likely benign/likely pathogenic and one with VUS, respectively. Exon 11 exhibited seven SNPs likely benign/ benign/likely pathogenic and three likely benign/benign. It also had three VUS with two amino acid changes followed by one likely benign. Exon 16 showed six SNPs likely benign, whereas exon 3 and exon 2 had one each of VUS and benign SNPs (two), respectively (Table 2). The SNPs of each exon percent of two germline genes, i.e., BRCA1 (36) and BRCA2 (36), are shown in Figures 3 and 4.

\section{Age at diagnosis and mutation frequency}

The average age was $47.3 \pm 3.2$ ranging from 23 to 72 years. The age group 41-60 years ( 18 patients) had a high frequency

Table I Patients with BRCAI and BRCA2 mutations

\begin{tabular}{|c|c|c|c|c|c|c|c|c|c|}
\hline $\begin{array}{l}\text { Pt. } \\
\text { No. }\end{array}$ & Location & Gene & $\begin{array}{l}\text { Age } \\
\text { (years) }\end{array}$ & c.dna & $\begin{array}{l}\text { Amino acid } \\
\text { change }\end{array}$ & $\begin{array}{l}\text { Mutation } \\
\text { type }\end{array}$ & F/S & $\begin{array}{l}\text { Mutation } \\
\text { effect }\end{array}$ & $\begin{array}{l}\text { Novel/ } \\
\text { Reference }\end{array}$ \\
\hline I & Exon 27 & BRCA2 & 56 & $c .9976 \mathrm{~A}>\mathrm{T}$ & p.Lys3326Ter & SNP & Familial & Nonsense & rsII57|833:a, b \\
\hline 2 & Exon 2 & BRCAI & 51 & c.66_67delAG & p.Glu23Val & Deletion & NA & Frameshift &,$- \mathrm{a}$ \\
\hline 3 & Exon 10 & BRCAI & 53 & c.3119G>A & p.Ser I040Asn & SNP & Sporadic & Missense & rs4986852:a, b \\
\hline 4 & Exon II & BRCAI & 49 & c. $24 \mid 2 \mathrm{G}>\mathrm{C}$ & p.Gln804His & SNP & Familial & Missense & rs5574654l:a, b \\
\hline 5 & Exon 6 & BRCA2 & 29 & c.484_484delG & p.Prol63fs & Deletion & Familial & Frameshift & Novel \\
\hline \multirow[t]{4}{*}{6} & Exon II & BRCA2 & 31 & c. $297 \mid \mathrm{A}>\mathrm{G}$ & p.Asn99।Asp & SNP & Familial & Missense & rsI799944:a, b \\
\hline & & & & c. $4779 A>C$ & p.Glu I593Asp & & & & rs80358703:a, b \\
\hline & Exon 16 & BRCAI & & c. $5019 \mathrm{G}>\mathrm{A}$ & p.Met I673lle & & & & rsI799967:b \\
\hline & Exon 10 & BRCA2 & & $c .865 \mathrm{~A}>\mathrm{C}$ & p.Asn289His & & & & rs766I73:a, b \\
\hline \multirow[t]{2}{*}{7} & Exon 16 & BRCAI & 23 & c.5019G $>A$ & p.Met I673lle & SNP & Sporadic & Missense & rsl799967:b \\
\hline & Exon 10 & BRCA2 & & $c .865 \mathrm{~A}>\mathrm{C}$ & p.Asn289His & & & & rs766I73:a, b \\
\hline 8 & Exon 10 & BRCAI & 39 & c. $31 \mid 3 A>G$ & p.Glu I038Gly & SNP & Sporadic & Missense & rsl694l:a, b \\
\hline 9 & Exon 09 & BRCAI & 45 & c. $2077 \mathrm{G}>\mathrm{A}$ & p.Asp693Asn & SNP & Familial & Missense & rs4986850:a, b \\
\hline \multirow[t]{6}{*}{10} & Exon II & BRCAI & 55 & c. $2612 \mathrm{C}>\mathrm{T}$ & p.Pro87I Leu & SNP & Familial & Missense & rs7999|7:a, b \\
\hline & Exon 10 & & & c. $31 \mid 3 \mathrm{~A}>\mathrm{G}$ & p.Glu 1038Gly & & & & rsI694I:a, b \\
\hline & Exon II & & & c. $3548 \mathrm{~A}>\mathrm{G}$ & p.Lys I I83Arg & & & & rsl6942:a, b \\
\hline & Exon 16 & & & c. $4837 \mathrm{~A}>\mathrm{G}$ & p.Ser I6I3Gly & & & &,$- \mathrm{a}$ \\
\hline & Exon II & BRCA2 & & c. $5744 \mathrm{C}>\mathrm{T}$ & p.Thr1915Met & & & &,$- a$ \\
\hline & Exon 14 & & & c.7397T >C & p.Val2466Ala & & & &,- a \\
\hline \multirow[t]{3}{*}{ II } & Exon 10 & BRCA2 & 63 & c. $1114 \mathrm{~A}>\mathrm{C}$ & p.Asn $372 \mathrm{His}$ & SNP & Familial & Missense & rs|44848:a, b \\
\hline & Exon II & & & c. $297 \mid A>G$ & p.Asn99|Asp & & & & rsI799944:a, b \\
\hline & Exon 10 & & & $c .865 \mathrm{~A}>\mathrm{C}$ & p.Asn289His & & & & rs766173:a, b \\
\hline 12 & Exon 18 & BRCA2 & 52 & $c .8117 A>G$ & p.Asn2706Ser & SNP & Familial & Missense & rs80359055:a, b \\
\hline \multirow[t]{2}{*}{13} & Exon II & BRCAI & 40 & c. $252 \mid \mathrm{IC}>\mathrm{T}$ & p.Arg84ITry & SNP & Sporadic & Missense & rs|800709:a, b \\
\hline & Exon 10 & BRCAI & & c. $3119 \mathrm{G}>\mathrm{A}$ & p.Ser I040Asn & & & & rs4986852:a, b \\
\hline \multirow[t]{2}{*}{14} & Exon II & BRCA2 & 49 & c. $297 \mid \mathrm{A}>\mathrm{G}$ & p.Asn99|Asp & SNP & Sporadic & Missense & rs I799944:a, b \\
\hline & Exon 10 & BRCA2 & & $c .865 \mathrm{~A}>\mathrm{C}$ & p.Asn289His & & & & rs766173:a, b \\
\hline 15 & Exon 10 & BRCA2 & 39 & $c .943 \mathrm{~T}>\mathrm{A}$ & p.Cys3।5Ser & SNP & Familial & Missense & rs7948320I:a, b \\
\hline \multirow[t]{6}{*}{16} & Exon 10 & BRCA2 & 59 & c. $1114 \mathrm{~A}>\mathrm{C}$ & p.Asn $372 \mathrm{His}$ & SNP & Familial & Missense & rs|44848:a, b \\
\hline & Exon II & BRCAI & & c. $2612 \mathrm{C}>\mathrm{T}$ & p.Pro87I Leu & & & & rs7999।7:a, b \\
\hline & Exon 10 & & & c. $31 \mid 3 A>G$ & p.Glu 1038Gly & & & & rsI694I:a, b \\
\hline & Exon II & & & c. $3548 A>G$ & p.Lys I I83Arg & & & & rsl6942:a, b \\
\hline & Exon 16 & & & c. $4837 \mathrm{~A}>\mathrm{G}$ & p.Ser 1613Gly & & & &,$- \mathrm{a}$ \\
\hline & Exon 14 & BRCA2 & & c. $7397 \mathrm{~T}>\mathrm{C}$ & p.Val2466Ala & & & &,$- a$ \\
\hline
\end{tabular}


Table I (Continued)

\begin{tabular}{|c|c|c|c|c|c|c|c|c|c|}
\hline $\begin{array}{l}\text { Pt. } \\
\text { No. }\end{array}$ & Location & Gene & $\begin{array}{l}\text { Age } \\
\text { (years) }\end{array}$ & c.dna & $\begin{array}{l}\text { Amino acid } \\
\text { change }\end{array}$ & $\begin{array}{l}\text { Mutation } \\
\text { type }\end{array}$ & F/S & $\begin{array}{l}\text { Mutation } \\
\text { effect }\end{array}$ & $\begin{array}{l}\text { Novel/ } \\
\text { Reference }\end{array}$ \\
\hline 17 & $\begin{array}{l}\text { Splice } \\
\text { site } 3\end{array}$ & BRCAI & 74 & $c .5|37+| G>A$ & - & & Familial & Missense & Novel \\
\hline \multirow[t]{2}{*}{18} & Exon II & BRCA2 & 25 & $c .6231 \mathrm{G}>\mathrm{C}$ & p.Lys2077Asn & SNP & Sporadic & Missense & rs54I826447:b \\
\hline & Exon 27 & & & $c .9976 \mathrm{~A}>\mathrm{T}$ & p.Lys3326Ter & & & Non sense & rsII57|833:a, b \\
\hline \multirow[t]{2}{*}{19} & Exon 10 & BRCA2 & 68 & c. $865 \mathrm{~A}>\mathrm{C}$ & p.Asn289His & & Sporadic & Missense & rs766I73:a, b \\
\hline & Exon 25 & & & c. $9380 \mathrm{G}>\mathrm{A}$ & p.Trp3127Ter & & & Nonsense & rs803592 I I:a, b \\
\hline \multirow[t]{6}{*}{20} & Exon 10 & BRCA2 & 67 & c. $I 1 \mid 4 A>C$ & p.Asn $372 \mathrm{His}$ & SNP & Familial & Missense & rs|44848:a, b \\
\hline & Exon II & BRCAI & & c. $26 \mid 2 C>T$ & p.Pro87ILeu & & & & rs799917:a, b \\
\hline & Exon 10 & & & $c .31 \mid 3 A>G$ & p.Glu I038Gly & & & & rsI694I:a, b \\
\hline & Exon II & & & c. $3548 \mathrm{~A}>\mathrm{G}$ & p.Lys I I83Arg & & & & rs16942:a, b \\
\hline & Exon 16 & & & c. $4837 A>G$ & p.Ser I6I3Gly & & & &,$- a$ \\
\hline & Exon 14 & BRCA2 & & c.7397T >C & p.Val2466Ala & & & &,$- a$ \\
\hline \multirow[t]{7}{*}{21} & Exon II & BRCAI & 49 & c. $2612 \mathrm{C}>\mathrm{T}$ & p.Pro87ILeu & SNP & Familial & Missense & rs7999|7:a, b \\
\hline & Exon II & BRCA2 & & c. $297 \mid A>G$ & p.Asn99|Asp & & & & rsI799944:a, b \\
\hline & Exon 10 & BRCAI & & c. $31 \mid 3 A>G$ & p.Glu I038Gly & & & & rsl694I:a, b \\
\hline & Exon II & & & c. $3548 \mathrm{~A}>\mathrm{G}$ & p.Lys I I83Arg & & & & rs16942:a, b \\
\hline & Exon 16 & & & c. $4837 A>G$ & p.Ser I6I3Gly & & & &,- a \\
\hline & Exon 14 & BRCA2 & & c.7397T >C & p.Val2466Ala & & & &,$- \mathrm{a}$ \\
\hline & Exon 10 & & & c. $865 \mathrm{~A}>\mathrm{C}$ & p.Asn289His & & & & rs766I73:a, b \\
\hline 22 & Exon 10 & BRCAI & 30 & c.3328_3330delAAG & p.Lys IIIOdel & Deletion & Familial & In-frame del. & rs80358335:a, b \\
\hline 23 & Exon 14 & BRCAI & 37 & c.4442_4442delG & p.Ser I 48Ifs & Deletion & Familial & Frameshift & NOVEL \\
\hline \multirow[t]{7}{*}{24} & Exon II & BRCAI & 72 & $c .2612 C>T$ & p.Pro87ILeu & SNP & Familial & Missense & rs7999|7:a, b \\
\hline & Exon II & BRCA2 & & c. $297 \mid A>G$ & p.Asn99|Asp & & & & rs|799944:a, b \\
\hline & Exon 10 & BRCAI & & c. $31 \mid 3 A>G$ & p.Glu I038Gly & & & & rsl694I:a, b \\
\hline & Exon II & & & c. $3548 \mathrm{~A}>\mathrm{G}$ & p.Lys II83Arg & & & & rs16942:a, b \\
\hline & Exon 16 & & & c. $4837 \mathrm{~A}>\mathrm{G}$ & p.Ser I6I3Gly & & & &,$- \mathrm{a}$ \\
\hline & Exon 14 & BRCA2 & & c.7397T >C & p.Val2466Ala & & & &,$- \mathrm{a}$ \\
\hline & Exon 10 & & & $c .865 \mathrm{~A}>\mathrm{C}$ & p.Asn289His & & & & rs766I73:a, b \\
\hline 25 & $\begin{array}{l}\text { Splice } \\
\text { site } 3\end{array}$ & BRCAI & 44 & $c .5|37+| G>A$ & - & & Sporadic & Missense & Novel \\
\hline \multirow[t]{7}{*}{26} & Exon II & BRCAI & 52 & c. $2612 \mathrm{C}>\mathrm{T}$ & p.Pro87ILeu & SNP & Sporadic & Missense & rs7999|7:a, b \\
\hline & Exon II & BRCA2 & & c. $297 \mid A>G$ & p.Asn99।Asp & & & & rsI799944:a, b \\
\hline & Exon 10 & BRCAI & & c. $31 \mid 3 A>G$ & p.Glu I038Gly & & & & rsI694I:a, b \\
\hline & Exon II & & & c. $3548 \mathrm{~A}>\mathrm{G}$ & p.Lys I I83Arg & & & & rs16942:a, b \\
\hline & Exon 16 & & & c. $4837 A>G$ & p.Ser I6I3Gly & & & &,$- \mathrm{a}$ \\
\hline & Exon 14 & BRCA2 & & c.7397T >C & p.Val2466Ala & & & &,$- \mathrm{a}$ \\
\hline & Exon 10 & & & c. $865 \mathrm{~A}>\mathrm{C}$ & p.Asn289His & & & & rs766173:a, b \\
\hline \multirow[t]{2}{*}{27} & Exon 03 & BRCA2 & 48 & c. $125 \mathrm{~A}>\mathrm{G}$ & p.Try42Cys & SNP & Familial & Missense & rs4987046:a, b \\
\hline & Exon II & & & c.3167_3170delAAAA & p.Gln 1056 & Deletion & & Frameshift & rs80359372:a, b \\
\hline 28 & Exon 25 & BRCA2 & 44 & c. $9380 \mathrm{G}>\mathrm{A}$ & p.Trp3127Ter & & Familial & Nonsense & rs803592I I:a, b \\
\hline \multirow[t]{3}{*}{29} & Exon II & BRCA2 & 57 & c. $297 \mid A>G$ & p.Asn99|Asp & SNP & Sporadic & Missense & rsI799944:a, b \\
\hline & & & & $c .5744 C>T$ & p.Thr I9I5Met & & & &,- a \\
\hline & Exon 10 & BRCA2 & & $c .865 \mathrm{~A}>\mathrm{C}$ & p.Asn289His & & & & rs766173:a, b \\
\hline 30 & Exon 10 & BRCA2 & 50 & c.2169_2170insA & p.Val726fs & Insertion & NA & Frameshift & Novel \\
\hline 31 & Exon II & BRCA2 & 53 & $c .5744 \mathrm{C}>\mathrm{T}$ & p.Thr I9I5Met & SNP & Familial & Missense & rs4987। I7:a, b \\
\hline \multirow[t]{2}{*}{32} & Exon 10 & BRCA2 & 39 & c. $11 \mid 4 \mathrm{~A}>\mathrm{C}$ & p.Asn372His & SNP & Familial & Missense & rs|44848:a, b \\
\hline & & BRCAI & & c. $2077 \mathrm{G}>\mathrm{A}$ & p.Asp693Asn & & & & rs4986850:a, b \\
\hline 33 & Exon II & BRCA2 & 34 & $c .4258 \mathrm{G}>\mathrm{T}$ & p.Asp I 420Tyr & SNP & Sporadic & Missense & rs28897727:a, b \\
\hline 34 & Exon II & BRCA2 & 55 & c. $4779 \mathrm{~A}>\mathrm{C}$ & p.Glu I593Asp & SNP & Sporadic & Missense & rs80358703:a, b \\
\hline 35 & Exon 10 & BRCAI & 67 & c. $2077 \mathrm{G}>\mathrm{A}$ & p.Asp693Asn & SNP & Sporadic & Missense & rs4986850:a, b \\
\hline
\end{tabular}

Notes: a, BIC; b, dbSNP; -, unknown; Familial $n=20$; Sporadic $n=13 ;$ NA $n=2$.

Abbreviations: F, familial; S, sporadic; BIC, Breast Cancer Information Core; dbSNP, single-nucleotide polymorphism database; NA, not available; Pt, patient. 


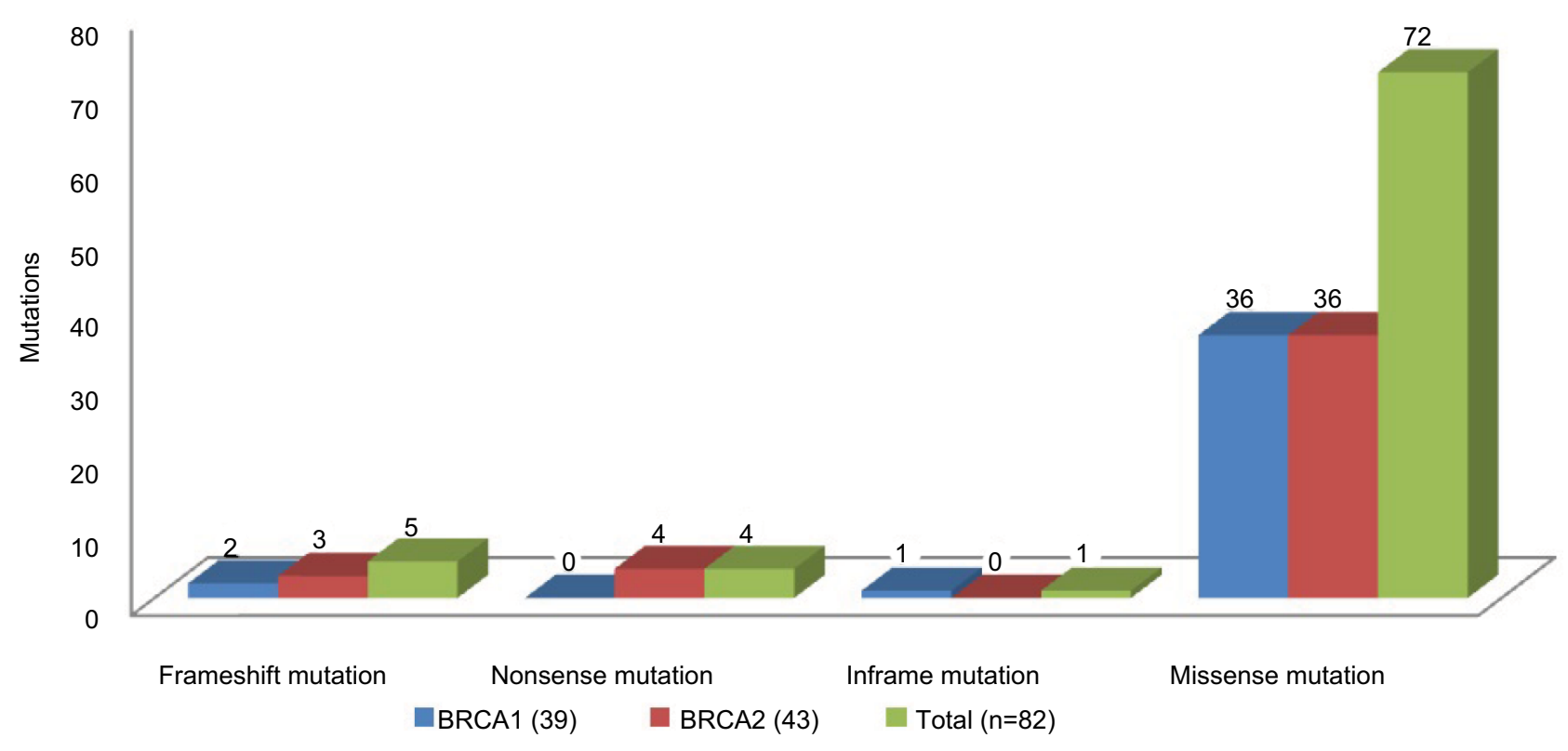

Figure 2 Mutation types in BRCAI and BRCA2 mutations.

of mutations (45/82: $54.9 \%$ ) followed by $>61$ years (six) with 20 (20/82: 24.4\%) and <40 years (11) with 17 (17/82: $20.7 \%$ ). However, the $23-60$ years group had 62 mutations (62/82: 75.6\%) (Figure 5).

\section{Discussion}

BC can be caused by germline mutations in BRCA1 and BRCA2, or by several other genes. In our cohort, we analyzed 79 referral cases using NGS and gene sequencing of Sanger with other reports of clinicians for phenotypic variations. Only 35 cases $(44.30 \%)$ detected positive for BRCA1 or BRCA2. The mutations in BRCA2 (52.4\%) were marginally higher than those in BRCA1 (47.6\%) in our study. Similar results were observed in a North Indian population where BRCA2 had maintained equal distribution of BRCA1/2. ${ }^{2}$ BRCA2 mutations contribute more than BRCA1 mutations in Philippine women in support of our data. ${ }^{17}$ Jang et al ${ }^{18}$ in Korean cases found no variations between BRCA1 and BRCA2 mutations. But in a South Indian population, BRCA1 mutations were more than BRCA2 germline mutations. ${ }^{19}$

Others included five frameshift mutations, where protein was truncated altering its structure and function. In these, three were novel types - BRCA2 (exon 6) of c.484-484delG, BRCA2 (exon 10) of c.2169_2170InsA and BRCA1 (exon 14) of c.4442_4442delG) - and the other two were referenced in BIC and the SNP database. All were pathogenic and familial in our study. Four nonsense mutations were found in our cancer patients, with two each of familial and sporadic in BRCA2 mutations, and these could lead to premature termination of protein due to change in specific amino acid proving lethal, and referenced in database. Furthermore, only one in-frame deletion in exon 10 of BRCA1(c.3328_3330delAAG) was detected in our cohort which was familial and deleterious. Finally, in our study, five novel mutations were detected, i.e., splice-site missense (two) and frameshift (three) types. Similar mutations are well reported globally in $\mathrm{BC}$ and ovarian cancer women except novel mutations in our report. ${ }^{12,18,19}$

High frequency of missense polymorphism (72) in women with $\mathrm{BC}$ is seen in our study, which was equal in both genes. Exons 10, 11 and 16 were more than exons 2, 3 and 9 of both genes, and all these may be responsible for $\mathrm{BC}$ in these patients depending upon amino acid substitution and its subsequent effect on protein structure and function. ${ }^{2} \mathrm{Few}$ missense polymorphic SNPs detected in Algerian populations were tested negative for both gene mutations, but could have a role as a susceptibility BC marker in BRCA1/2. ${ }^{20}$ In both familial (20) and sporadic (15) cases, few expressed multiples of polymorphic sites as found by others. ${ }^{20}$ In these mutations, two were found to be novel (two splice sites) in BRCA1 only, i.e., c.5137+1G $>\mathrm{A}$ in two cases in which $\mathrm{G}$ was replaced by $\mathrm{A}$, and others were registered. In our cohort, as per database of ClinVar (National Center for Biotechnology Information [NCBI]), dbSNP and BIC revealed most of them were benign in BRCA1 except two splice site types (c.5137+1G>A) which were pathogenic (No. 8, 11 of Table. 1) and one with VUS (p.A841T). In BRCA2, five SNPs, p.Cys315Ser, p.Gly1593Asp(2), p.Lys2077Asn and p.Asn2106Ser were with VUS and others in both genes, and were benign only. 
Table 2 Types of polymorphic SNPs in BRCAI and BRCA2

\begin{tabular}{|c|c|c|c|c|}
\hline \multicolumn{5}{|c|}{ BRCAI } \\
\hline $\begin{array}{l}\text { Serial } \\
\text { No. }\end{array}$ & $\begin{array}{l}\text { Exon } \\
\text { No. }\end{array}$ & $\begin{array}{l}\text { Amino acid } \\
\text { change }\end{array}$ & Frequency & Effect \\
\hline \multirow[t]{3}{*}{ I } & Exon 10 & p.Glu I038Gly & 7 & Benign \\
\hline & & p.Asp693Asn & 2 & Likely pathogenic \\
\hline & & p.Ser 1040Asn & 2 & VUS \\
\hline \multirow[t]{4}{*}{2} & Exon II & p.Pro87ILeu & 6 & Likely benign \\
\hline & & p.Lys I I83Arg & 6 & Likely benign \\
\hline & & p.Gln804His & I & VUS \\
\hline & & p.Arg84ITyp & I & VUS \\
\hline \multirow[t]{2}{*}{3} & Exon 16 & p.Ser 16I3Gly & 6 & Benign \\
\hline & & p.Met I673lle & 2 & VUS \\
\hline \multirow[t]{3}{*}{4} & Exon 9 & p.Asp693Asn & 1 & Likely benign \\
\hline & & $C .5137+\mid G>A$ & 2 & Pathogenic \\
\hline & & (Splice site*) & & \\
\hline \multicolumn{5}{|c|}{ BRCA2 } \\
\hline $\begin{array}{l}\text { Serial } \\
\text { No. }\end{array}$ & $\begin{array}{l}\text { Exon } \\
\text { No. }\end{array}$ & $\begin{array}{l}\text { Amino acid } \\
\text { change }\end{array}$ & Frequency & Effect \\
\hline \multirow[t]{6}{*}{1} & Exon 10 & p.Asn289His & 9 & Likely benign/ \\
\hline & & & & Benign/Likely \\
\hline & & & & pathogenic \\
\hline & & p.Asn $372 \mathrm{His}$ & 4 & Likely benign/ \\
\hline & & & & Likely pathogenic \\
\hline & & p.Cys3I5Ser & 1 & VUS \\
\hline \multirow[t]{8}{*}{2} & Exon II & p.Asn99| Asp & 7 & Likely benign/ \\
\hline & & & & Benign/Likely \\
\hline & & & & pathogenic \\
\hline & & p.Thr I9I5Met & 3 & Likely benign/ \\
\hline & & & & Benign \\
\hline & & p.Glu I593Asp & 2 & VUS \\
\hline & & p.Lys2077Asn & 1 & VUS \\
\hline & & p.Asp I 420Tyr & I & Likely benign \\
\hline 3 & Exon 16 & p.Val2466Ala & 6 & Likely benign \\
\hline 4 & Exon 2 & p.Asn2706Ser & 1 & VUS \\
\hline 5 & Exon 3 & p.Tyr42Cys & 1 & Benign \\
\hline
\end{tabular}

Notes: *Missense of splice site types leading protein termination and novel. BRCAI $=36 \mathrm{SNPs}$; BRCA2 = $36 \mathrm{SNPs}$. Effect is as per ClinVar, NCBI and BIC databases.

Abbreviations: VUS, variant of unknown significance; SNP, single-nucleotide polymorphism; NCBI, National Center for Biotechnology Information; BIC, Breast Cancer Information Core.

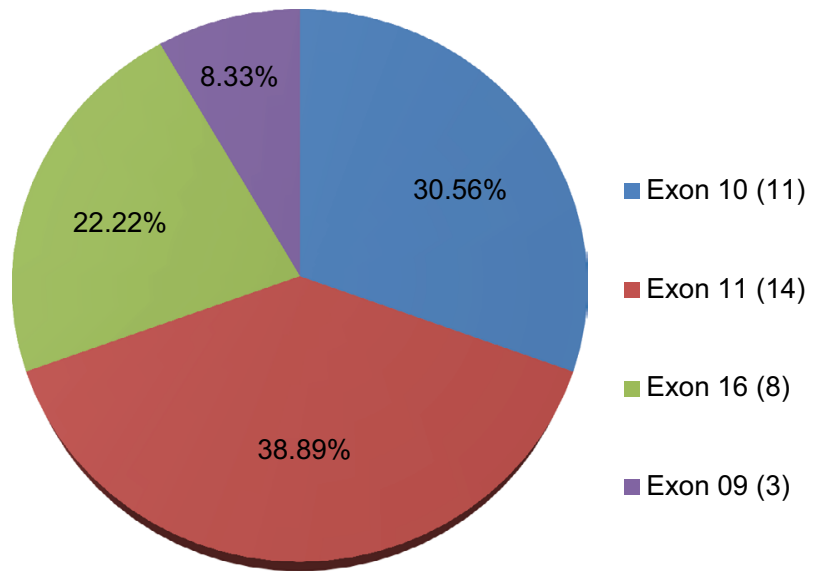

Figure 3 BRCAI SNP mutations. Abbreviation: SNP, single-nucleotide polymorphism.

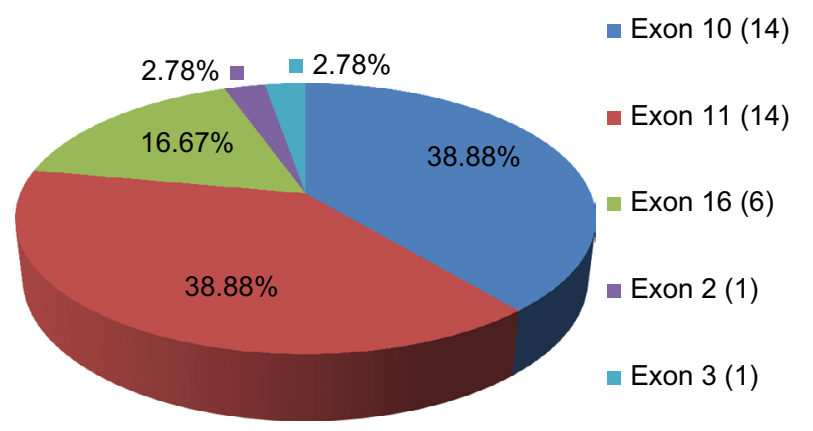

Figure 4 BRCA2 SNP mutations.

Abbreviation: SNP, single-nucleotide polymorphism.

However, these can be confirmed further by other predictive programs such as POLYPHEN 2, SIFT and PROVEN ${ }^{20}$ as in silico analysis may not provide the pathogenicity of them. However, controversial reports exist about the role of SNPs in these BRCA1/2 genes for the induction of human breast and ovarian cancer (HBOC). ${ }^{2,18,21,22,23}$ Dombernowsky et $\mathrm{a}^{22}$ reported that missense mutations cannot explain the risk of HBOC in women with familial history. Contrarily, Dodova et $\mathrm{al}^{21}$ in their study implicated the role of missense variants which may be deleterious. In an Iranian population, Neamatzadeh et $\mathrm{al}^{23}$ detected these polymorphic SNPs at higher frequency in BRCA1 and BRCA2 genes compared to control subjects supporting our data. Valarmathi et $\mathrm{al}^{7}$ in their study indicated that many of these variants are proposed to be deleterious in the Indian population. We further propose that polymorphic sites in BRCA1 and BRAC2 genes to cause cancer are dependent on the frequency and nature of amino acid replaced in the protein in regard to their lethality. These SNPs of BRCA1/2 could be related to the pathological condition of $\mathrm{BC}$ patients which differ from pathogenic, likely pathogenic, benign and likely benign, VUS or mixed types. It requires further elucidation by analyzing BRCA1/2 haplotypes in relation to gene mutations. ${ }^{24}$

In our study, young age groups (23-60 years) had more frequency of mutations $62 / 82(75.6 \%)$. This indicated that age is also one of the factors to be noted including other lifestyles, ethnicity and geographic distribution for these differences in their frequency as reported earlier. ${ }^{25,26,27}$ Mannan et $\mathrm{al}^{12}$ found in their cohort, that the mutation rate was proportionally higher in patients aged $<50$ years. Wong et $\mathrm{al}^{26}$ reported that early age is to be considered for higher rate of BRCA gene variants in $\mathrm{HBOC}$ patients in support of our observations. In the Indian population, the proportion of patients with early onset of mutations to $\mathrm{BC}$ is also much higher than in Caucasians. In North India, ${ }^{2}$ lower BRCA mutations in early age were reported in comparison with other 


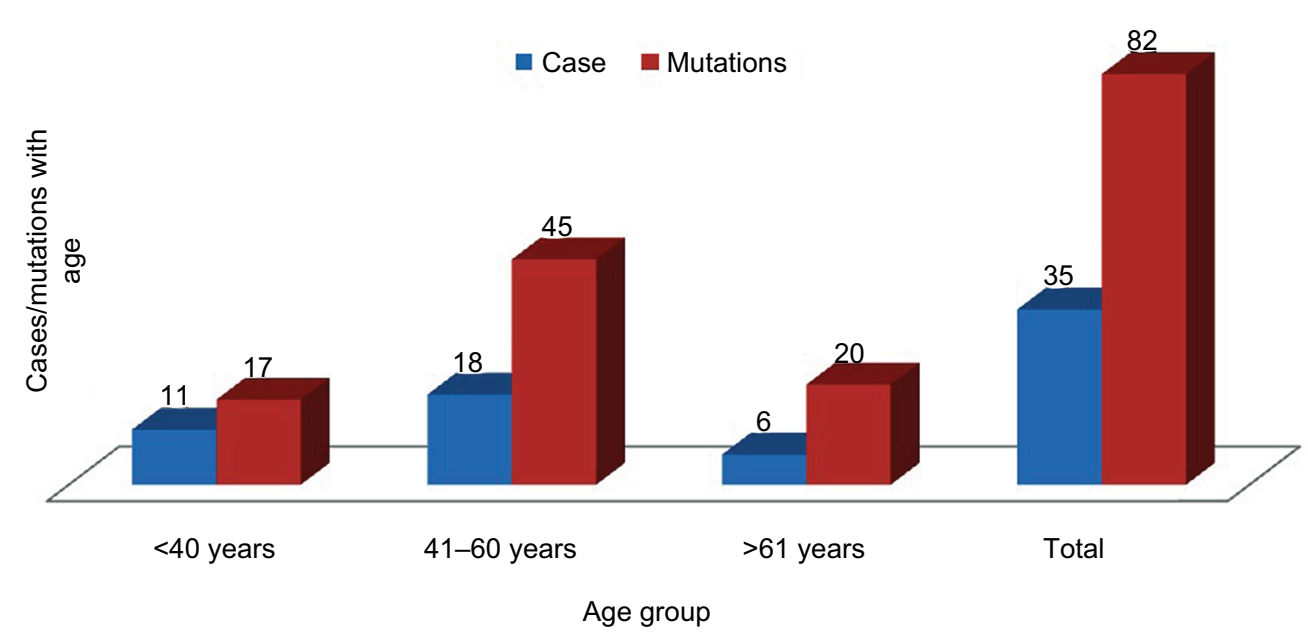

Figure 5 Cases and mutations in age groups of our study.

ethnic populations. Vaidyanathan et $\mathrm{al}^{8}$ detected a high rate of germline mutations with early age, like that of Mannan et al ${ }^{12}$ and our report. This discrepancy in these cohorts is related to the latest technologies including the gene panel used for monitoring gene mutations profiles in Indian patients.

In conclusion, BRCA2 gene mutations were marginally more than BRCA1 mutations. Young age groups had high frequency mutations which were also positively comparable. Polymorphic SNPs were higher than others, with five novel mutations, i.e., three frameshift and two spice site types, where synthesis of premature or truncating proteins occurs by amino acid replacement for their termination, respectively. Nonsense, in-frame and frameshift with splice site were pathogenic, and other polymorphic SNPs were benign, pathogenic and variant of unknown significance. The association of these mutations with haplotypes of BRCA1/2 needs to be evaluated in our future work in addition to age. This may help understand the role of SNPs in the causation of $\mathrm{BC}$ for better treatment and genetic testing highlighting sporadic and familial types in the Indian population.

\section{Acknowledgments}

The authors are thankful to all the staff including the clinicians of Supratech Micropath Laboratory, Ahmedabad, India, for their continuous assistance in this work. Parth S Shah, Nidhi D Shah, Sandip C Shah and Bhavini S Shah are son, daughter-in-law, and father and mother, respectively, and run this research institute.

\section{Author contributions}

Drs Nidhi D Shah and Parth S Shah contributed to writing the results and discussion during manuscript preparation when they visited India. Hari Shankar P Ray and Yash Y Panchal were involved in the collection of blood from the patients after duly completed consent forms, blood analysis, DNA extraction, DNA sequencing, and data analysis of 79 patients. Dr Sandip C Shah, Dr Bhavini S Shah and Dr Mandava V Rao contributed to the preparation of reports after finalization of the results and the final preparation of the manuscript for submission to the journal. All authors contributed toward data analysis, drafting and critically revising the paper and agree to be accountable for all aspects of the work.

\section{Disclosure}

The authors report no conflicts of interest in this work.

\section{References}

1. Rajkumar T, Soumittra N, Nancy KN, Shanta V. BRCA 1, BRCA 2 and CHEK2 (1100 del C) germline mutations in hereditary and ovarian cancer families in South India. Asian Pac J Can Prev. 2003;4: 203-208.

2. Saxena S, Chakraborty A, Kaushal M, et al. Contributions of germline $B R C A 1$ and $B R C A 2$ sequence alterations to breast cancer in Northern India. BMC Med Gen. 2006;7:75.

3. Indian Council of Medical Research (ICMR) News. Over 17 lakh new cancer cases in India by 2020. 2016. Available from: http://www. mid-day.com/articles.

4. Miki Y, Swensen J, Shattuck-Eidens D, et al. A strong candidate for the breast and ovarian cancer susceptibility gene BRCA1. Science. 1994;266:66-71.

5. Shattuck-Eidens D, McClure M, Simard J, et al. A collaborative survey of 80 mutations in BRCA, breast and ovarian cancer susceptibility gene. Implications for presymptomatic testing and screening. JAMA. 1995;273:535-541.

6. Shattuck-Eidens D, Oliphant A, McClure M, et al. BRCA1 sequence analysis in woman at high risk for susceptibility mutations. JAMA. 1997;278:1242-1250.

7. Valarmathi MT, Sawhney M, Deo SS, Shukla NK, Das SN. Novel germline mutations in the BRCA1 and BRCA2 genes in Indian breast and ovarian cancer families. Hum Mutat. 2004;23:205-215. 
8. Vaidyanathan K, Lakhotia S, Ravishankar HM, Tabassum U, Mukherjee G, Somasundaram K. BRCA1 and BRCA2 germline mutation analysis among Indian woman from south India: identification of four novel mutations and high frequency occurrence of 185 del.AG mutations. J Biosci. 2009;34:415-422.

9. Karami F, Mehdipour P. A comprehensive focus on global spectrum of BRCA1 and BRCA2 mutations in breast cancer. Biomed Res Int. 2013;2013:928562.

10. Ferlay J, Soerjomataram I, Dixshit R, et al. Cancer incidence and mortility worldwide: sources, methods and major patterns in GLOBOCAN 2012. Int J Cancer. 2015;136. E359-386.

11. Alteri R, Bertaut T, Brinton LA, et al on behalf of the American Cancer Society. Breast cancer facts and figures 2015-2016. Atlanta, GA: American Cancer Society. 2015;1-38.

12. Mannan AU, Singh J, Lakshmikeshava R, et al. Detection of high frequency mutations in a breast and ovarian cancer cohort: implications of embracing a multigene panel in molecular diagnosis in India. J Hum Genet. 2016;61:515-522.

13. Torre LA, Bray F, Siegel RL, Ferlay J, Lortet-Tieulent J, Jemal A. Global cancer statistics, 2012. CA Cancer J Clin. 2015;65:87-108.

14. Jemal A, Center MM, DeSantis C, Ward EM. Global patterns of cancer incidence and mortality rates and trends. Cancer Epidemiol Biomarkers Prev. 2010;19(8):1893-1907.

15. Landrum MJ, Lee JM, Riley GR, et al. ClinVar: public archive of relationships among sequence variation and human phenotype. Nucleic Acids Res. 2014;42:980-985.

16. Abecasis GR, Auton A, Brooks LD, et al on behalf of the 1000 Genomes Project Consortium. An integrated map of genetic variation from 1,092 human genomes. Nature. 2012;491:56-65.

17. De Leon Matsuda ML, Liede A, Kwan E, et al. BRCA1 and BRCA2 mutations among breast cancer patients from the Philippines. Int $J$ Cancer. 2002;98:596-603.
18. Jang JH, Lee JE, Kwon MJ, et al. Spectra of BRCA1 and BRCA2 mutations in Korean patients with breast cancer: the importance of whole gene sequencing. J Hum Genet. 2012;57:212-215.

19. Soumittra N, Meenakumari B, Parija T, et al. Molecular genetics analysis of hereditary breast and ovarian cancer patients in India. Heredi Clin Pract. 2009;7:13.

20. Cherbal F, Salhi N, Bakour R, Adane S, Boualga K, Maillet P. BRCA1 and BRCA2 unclassified variants and missense polymorphisms in Algerian breast/ovarian cancer families. Dis Markers. 2012;32:343-353.

21. Dodova RI, Mitkova AV, Dacheva DR, et al. Spectrum and frequencies of BRCA1/2 mutations in Bulgarian high risk breast cancer patients. BMC Cancer. 2015;15:523.

22. Dombernowsky SL, Weischer M, Freiberg JJ, Bojesen SE, TybjaergHansen A, Nordestgaard BG. Missense polymorphisms in BRCA1 and BRCA2 and risk of breast and ovarian cancer. Cancer Epidemiol Biomarkers Prev. 2009;8:2339-2342.

23. Neamatzadeh H, Shiryazdi SM, Kalantar SM. BRCA1 and BRCA2 mutations in Iranian breast cancer patients: A systematic review. J Res Med Sci. 2015;20:284-293.

24. Judkins T, Hendrickson BC, Deffenbaugh AM, et al. Application of embryonic lethal or other obvious phenotypes to characterize the clinical significances of genetic variants found in trans with known deleterious mutations. Cancer Res. 2005:65:10096-10103.

25. Gholipoorfeshkecheh R, Arjunan S. Genotyping frequent BRCA1 SNPs in familiar breast cancer in Indian population by restriction fragment length polymorphism and sequencing. Adv Appl Sci Res. 2014;5:262-267.

26. Wong YE, Yhenkav S, Chan THC, et al. Predictive factors for BRCA1 and BRCA2 genetic testing in an Asian based population. PLoS One. 2015;10:1-12.

27. Sharma S, Rajaram S, Sharma T, et al. Role of BRCA1 and BRCA2 gene mutations in epithelial ovarian cancer in Indian population: a pilot study. Int J Biochem Mol Biol. 2014;5(1):1-10.
The Application of Clinical Genetics

\section{Publish your work in this journal}

The Application of Clinical Genetics is an international, peer-reviewed open access journal that welcomes laboratory and clinical findings in the field of human genetics. Specific topics include: Population genetics; Functional genetics; Natural history of genetic disease; Management of genetic disease; Mechanisms of genetic disease; Counselling and ethical
Dovepress

issues; Animal models; Pharmacogenetics; Prenatal diagnosis; Dysmorphology. The manuscript management system is completely online and includes a very quick and fair peer-review system, which is all easy to use. Visit http://www.dovepress.com/testimonials.php to read real quotes from published authors. 